\title{
Identification Precision of Vulnerability to Poverty Indexes: Does Information Quantity Matter?
}

\author{
Martina Celidoni • Isabella Procidano
}

Accepted: 12 April 2014/Published online: 3 May 2014

(C) Springer Science+Business Media Dordrecht 2014

\begin{abstract}
Vulnerability to poverty has been proposed in the literature as an ex ante measure of poverty risk useful for the identification of those who may fall into poverty in the future (Zhang and Guanghua 2008). This paper complements the existing literature on vulnerability measures, by investigating empirically how indexes precision varies according to the quantity of information available, in order to understand which is the best predictor of poverty conditional on data at hand. Using the British Household Panel Survey, we show that information quantity affects differently the predictive power of indexes.
\end{abstract}

Keywords Poverty $\cdot$ Risk $\cdot$ Vulnerability $\cdot$ Receiver operating characteristic (ROC) curve

\section{Introduction}

According to a recent report (OECD 2011) on income inequality, the gap between the rich and the poor in OECD countries had widened continuously over the nineties until

\footnotetext{
This paper was partly based on work carried out during a visiting to the European Centre for Analysis in the Social Sciences (ECASS) at the Institute for Social and Economic Research, University of Essex and supported by the Access to Research Infrastructures action under the EU Improving Human Potential Programme. Data from the British Household Panel Survey (BHPS) were supplied by the UK Data Archive. Neither the original collectors of the data nor the archive bear any responsibility for the analysis or interpretations presented here.

M. Celidoni $(\bowtie)$

Department of Economics and Management 'Marco Fanno', University of Padua, Via del Santo 33, 35123 Padua, Italy

e-mail: martina.celidoni@unipd.it

I. Procidano

Department of Management, Ca' Foscari University of Venice, Cannaregio 873,

Fondamenta San Giobbe, 30121 Venice, Italy

e-mail: isabella@unive.it
} 
nowadays when the global economic crises accelerated and exacerbated disparities by squeezing incomes from work and capital in most countries. Tax-benefit systems, together with fiscal stimuls policies, only partly have been able to mitigate welfare drops and alleviate some of the pain. But, since the economic crisis consequences tend to persist over time, the increasing recognition of the possibility that the most vulnerable individuals could be hit harder, gained the attention of governments, researchers and foundations on economic risk and its role as threat.

In the economic literature there are two concepts related to economic hazard: economic insecurity and vulnerability to poverty. Even if the two have evolved quite independently, they share a common basis. Both deal with an economic risk that produces anxiety (Osberg 1998) and represents a threat (Dercon 2006), but, according to Osberg (2010), they differ in terms of countries analysed, perspective and risk exposure consequences.

One of the main difference is that economic insecurity concerns more the ex post subjective measurement of the lack of safety rather than an objective poverty danger as vulnerability to poverty does. Everyone could feel economically insecure but only some individuals, those vulnerable, are likely to become poor in the future. Since vulnerability aims to identify the poor in advance it represents an ex ante information source for policies design that can be used for targeting purposes. Therefore, if the interest is to provide information for refining targeting strategies, vulnerability to poverty is the concern.

Chaudhuri et al. (2002), for example, write that what really matters for forward-looking anti-poverty interventions is vulnerability to poverty. Zhang and Guanghua (2008) argue that measuring vulnerability is important because it allows the identification of those who are not currently poor but may fall into poverty. Vulnerability therefore can be used, once vulnerable individuals are identified, to design appropriate policies to prevent them from falling into poverty. Also Jamal (2009), by highlighting the distinction between ex ante poverty prevention and ex post poverty alleviation interventions, considers vulnerability assessments as a way to improve risk-management policies.

In the literature there are several vulnerability to poverty measures, all focus on different and equally relevant aspects of poverty risk and are rich in terms of information summarised. It is not possible to distinguish a priori which is the best predictor of poverty, since they favour different sides of the same phenomenon. However, it is possible to assess their predictive power empirically, looking at some precision criteria. Borrowing from Celidoni (2013), this paper tries to evaluate vulnerability indexes peformances in anticipating poverty, conditional on information available to policy-makers. Especially we are interested in understanding if Celidoni (2013)'s findings, in terms of ranking among indexes, vary according to the number of income observations available for the same household. It could be in fact that some indexes are more precise than other the higher the quantity of information at hand. If this is the case, the choice among vulnerability measures, for targeting refinements, will depend also on the type of information that policymakers have available.

In comparing vulnerability indexes precision, we will take into account also targeting errors. The literature on targeting performance usually distinguishes between two indicators, leakage and undercoverage, to evaluate design issues (Muller and Bibi 2010). Under imperfect targeting, two types of error can be done. On one hand, one might fail to reach some of the poor individuals (undercoverage or type I error), this type of issue, as Atkinson (1995) noted, creates horizontal inefficiency compared to perfect targeting. On the other hand, benefits could be awarded to non-poor people, and this represents the type II error, whose monetary couterpart is defined as leakage. Both errors cannot be reduced at the same time. But, it can be argued that type I error is more relevant, compared to type II, 
when assessing indexes performances: identifying as non-vulnerable someone that will be poor in fact is worse that defining as vulnerable someone who will not be poor. We therefore present our results also controlling for low levels of undercoverage.

The outline of the paper is the following: Sect. 2 reviews the literature, Sect. 3 presents the data, Sect. 4 discusses the results and Sect. 5 concludes.

\section{Literature Review}

\subsection{Vulnerability to Poverty}

According to World Bank definition (2011), vulnerability to poverty is the probability, today, of being in poverty or to fall into deeper poverty in the future. Vulnerability is very different from the standard analysis of poverty because it recalls an ex ante perspective rather than an ex post assessment, allowing the design of protection policies that can prevent households and individuals from experiencing welfare losses.

The concept of vulnerability to poverty stems its roots in a seminal article by Jalan and Ravaillon (1998) on transient and chronic poverty. Here the authors noticed how in rural China variability in consumption accounts for a large part of the observed poverty: half of the mean squared poverty gap and over a third of the mean poverty gap is transient and directly attributable to year-to-year consumption fluctuations.

While theoretically vulnerability to poverty is almost well-defined as the risk of experiencing poverty, three different definitions can be recognised at the empirical level: vulnerability as expected poverty (VEP), vulnerability as low expected utility (VEU) and vulnerability as uninsured exposure to risk (VER). These definitions are all equally used in literature, since they describe the poverty risk according to three different perspectives.

The very first VEP version translates vulnerability into a probability measure of facing poverty in the future. More precisely, when welfare is defined in terms of consumption or income, then vulnerability of the $h$-th household (or individual), at time $t$, is $V_{h t}$, the probability that consumption or income tomorrow, $y_{h, t+1}$, falls below the poverty line, $z$, that is

$$
V_{h t}=\operatorname{Pr}\left(y_{h, t+1}<z\right) .
$$

Ligon and Schechter (2003) proposed a different measure, based on utility, to take properly into account risk sensitivity. They pointed out that a policy-maker, who allocates resources to minimise the expected value of one of the Foster et al. (1984) (FGT) indexes, would tend to assign too much risk to poorer households. Therefore they defined vulnerability as the difference between the utility derived from some level of certainty-equivalent, $z_{C E}$, at and above which the household $h$ would not be considered vulnerable, and the expected utility of consumption, $c_{h}$,

$$
V_{h}=U_{h}\left(z_{C E}\right)-E U_{h}\left(c_{h}\right) .
$$

This approach, while appealing in terms of risk considerations, has some drawbacks since it is necessary to specify a utility functional form for $U_{h}$ and a value for the risk aversion parameter. VEU has been used less extensively compared to VEP because it measures vulnerability in terms of utility units, with a less straightforward interpretation of the results.

The third approach, VER, even if based on intertemporal variability of consumption as VEP and VEU, is very different in terms of perspective: VER is backward-looking. VER is 
in fact an ex post assessment of the extent to which a negative income shock caused a welfare loss in terms of consumption. This third approach is based on the consumption smoothing and risk sharing literature, where the degree of vulnerability is defined by the extent to which the growth rate of household consumption covaries with the household income growth rate (Skoufias and Quisumbing 2003; Gerry and Li 2010). VER aims to understand if households are able to mitigate the effects of income shocks through formal or informal insurance strategies: if consumption and income are correlated, then the households use ineffective risk management instruments, increasing their vulnerability to negative income shocks. Using the following equation

$$
\Delta c_{h, t, v}=\beta \Delta \operatorname{lny}_{h, t, v}+\delta X_{h, t, v}+\sum_{t, v} \delta_{t, v} D_{t, v}+\Delta \varepsilon_{h, t, v},
$$

where $\Delta c_{h, t, v}$ denotes the growth rate from $t-1$ to $t$ of the total consumption of household $h$ in the community $v, \Delta l n y_{h, t, v}$ is the growth rate of income, $X$ is a vector of household characteristics, $D_{t, v}$ are other community or time varying controls and $\Delta \varepsilon_{h, t, v}$ is a household-specific error term, the parameter of interest for VER is $\beta$.

In this analysis, since we are interested in the ability of vulnerability measures to identify in advance the future poor, we will focus on the first approach mentioned, VEP, that has a forward-looking perspective and is easier to interpret.

Vulnerability to poverty has been often studied in developing countries (see among others Gaiha and Imai 2008; Imai et al. 2009; Jha et al. 2009; Gaiha et al. 2011) because poverty risk is, in relative terms, quantitatively more important, but volatile incomes are commonly encountered also in developed countries and are, under certain conditions, symptoms of being prone to poverty. Bandyopadhyay and Cowell (2007) for instance estimate vulnerability to poverty for the United Kingdom using the VER approach and quantile regressions. They found that, apart from those households around the poverty line, there are some, well away from the poverty zone, that are susceptible to be income shocks vulnerable.

In this paper we will use data drawn from the British Household Panel Survey (BHPS), the choice is driven mainly by the quality of data necessary to estimate properly vulnerability and to highlight the role of different available information sets. To emphasise the features of each index a sufficiently long longitudinal component is needed and information on the household disposable income has to be collected accurately. The BHPS meets all these requirements.

\subsection{Measuring Vulnerability as Expected Poverty}

In this subsection, we present the vulnerability to poverty measures of Table 1 that we will compare empirically in terms of predicting power using BHPS data. More precisely the estimated indexes are those proposed by Pritchett et al. (2000) and Chaudhuri et al. (2002) $(P C)$, Chaudhuri (2003) (C), Christiaensen and Boisvert (2000) (FGT1 and FGT2), Calvo and Dercon (2005) (CD rel and CD abs) and Dutta et al. (2011) (DFM1 and DFM2). We now describe them in detail. One of the first papers formalising the idea of a measure that can predict poverty is Pritchett et al. (2000), where the authors point out how many households, not currently in poverty, are vulnerable to events such as jobloss, or unexpected expenditures due to illnesses or economic downturns.

According to Pritchett et al. (2000), vulnerability is a risk measured in terms of probability that the expected income falls the poverty line, $z ; I[\cdot]$ is an indicator function that 
Table 1 Vulnerability to poverty indexes

\begin{tabular}{lll}
\hline Author(s) & Year & Index \\
\hline PC & 2000 & $V_{h t}=I\left[\operatorname{Pr}\left(\ln y_{h, t+1}<z \mid x_{h, t}\right)>p\right]$ \\
C & 2003 & $V_{h t}=\operatorname{Pr}\left(\ln y_{h, t+1}<z \mid x_{h, t}\right)$ \\
FGT & 2003 & $V_{h t}=\sum_{s}^{S} p_{s} I\left[y_{h, t+1}, z\right] \cdot\left[\left(z-y_{h, t+1}\right) / z\right]^{\alpha}, \quad \alpha=1,2$, \\
CD rel & 2005 & $V_{\alpha}=1-E\left[\left(\frac{\min \left(y_{h}, z\right)}{z}\right)^{\alpha}\right]$ \\
CD abs & 2005 & $V_{\beta}=E\left[\frac{e^{\beta\left(1-x_{h}\right)}-1}{e^{\beta}-1} x_{h}=\frac{\min \left(y_{h}, z\right)}{z}\right.$ \\
DFM1 & 2010 & $V_{h t}=\sum_{s=1}^{n} p_{s}\left(R\left(z, y_{h, t}\right)-y_{h, t+1}^{s}\right)^{\gamma}, \quad R\left(z, y_{h, t}\right)=z^{1-\alpha} y_{h, t}^{\alpha}$ \\
DFM2 & 2010 & $V_{h t}=\sum_{s=1}^{n} p_{s}\left(R\left(z, y_{h, t}\right)-y_{h, t+1}^{s}\right)^{\gamma}, \quad R\left(z, y_{h, t}\right)=z^{1+\alpha} \backslash y_{h, t}^{\alpha}$ \\
\hline
\end{tabular}

PC = Pritchett et al. (2000) and Chaudhuri et al. (2002); $\mathrm{C}=$ Chaudhuri (2003); FGT = Christiaensen and Boisvert (2000) that recalls Foster et al. (1984)'s index, FGT1 refers to FGT when $\alpha=1$ whereas FGT2 refers to FGT when $\alpha=2$; CD = Calvo and Dercon (2005); DFM = Dutta et al. (2011)

translates vulnerability into a state variable, by defining a probability threshold, $p$. The authors observe that everybody face a certain degree of poverty risk, also the richest individuals, therefore, to have a more reliable aggregate measure of poverty risk, called Headcount Vulnerable to Poverty Rate, they introduce the function, $I[\cdot]$, that takes value 1 if the probability computed is higher than 0.5 , and zero otherwise.

As already noticed in literature this approach fails to consider explicitly the depth of poverty (Hoddinott and Quisumbing 2003), but this issue has been adressed in Christiaensen and Boisvert (2000). Christiaensen and Boisvert (2000)'s index echoes the Foster et al. (1984)'s (FGT) index of poverty, where $\alpha$ is the relative weight attached to extreme poverty (FGT1 and FGT2 are computed with $\alpha$ respectively equal to 1 and 2), $S$ are contingences that the individual faces, and $p_{s}$ is the probability that the $s$-th state occurs, finally $I[\cdot]$ is a function that allows to consider only those states in which the expected income, $y_{h, t+1}$, falls below the poverty line $z$. According to Kamanou and Morduch (2002), a possible drawback, when using this index, is that it does not consider properly the persistency of the phenomenon.

Despite the discussed limitations, vulnerability expressed in terms of probability has been widely used because easy to interpret, even if very demanding in terms of data when translated empirically. Estimating Pritchett et al. (2000)'s vulnerability in fact requires some assumptions: to compute probabilities, information about the distribution of income or consumption is needed not only at the aggregate level but also at the household (or individual) level. This is the reason why in the empirical applications, to preserve computational simplicity, the distribution is always assumed to be normal (see among others Azam and Imai 2009; Zhang and Guanghua 2008; Jha et al. 2009; Chaudhuri et al. 2002, Gaiha et al. 2011). As the majority of restrictions are imposed by the empirical analysis, part of the literature on VEP has focused on overcoming the data limitations by improving the estimates of income or consumption variability. Chaudhuri et al. (2002) and Chaudhuri (2003), for instance, using the same measure of Pritchett et al. (2000), compute vulnerability when only cross-sectional information is available, with a consistent estimate of the variance. 
Following an axiomatic approach, Calvo and Dercon (2005) proposed other two classes of measures, that satisfy some additional properties to incorporate alternative risk aversion attitudes, more consistent with the empirical evidence. $C D$ rel satisfies the constant relative risk sensitivity, i.e. the efficiency loss due to risk is determined as a constant proportion of expected outcome. $C D$ abs meets instead the constant absolute risk sensitivity, i.e. the efficiency loss is a constant value of $y^{c e}-\hat{y}_{t+1}$, where $y^{c e}$ is the certainty-equivalent outcome. While different risk attitudes are the main novelty proposed by Calvo and Dercon (2005) in measuring vulnerability, Dutta et al. (2011) have recently highlighted the importance of current living standard in this context, by proposing two indexes. The authors argue that the threat of poverty depends not only on the poverty line, but also on the current living standard that can exacerbate or mitigate against the welfare loss; rather than a general poverty line $z$, they propose therefore an index of vulnerability based on an individual reference line $R\left(z, y_{h, t}\right)$. Moreover, their measure is flexible enough to account for two opposite effects of the current living standard, positive or negative. The index $D F M 1$ considers a reference line $R\left(z, y_{h, t}\right)$ that reflects the idea of worse consequences in term of vulnerability for those with higher current living standard, while $D F M 2$, on the contrary, accounts for the fact that low current income might exacerbate the potential drops in welfare. ${ }^{1}$ In this analysis, the vulnerability index proposed by Kamanou and Morduch (2002) is not considered because defined directly at the society level. Their approach therefore does not aim to identify the vulnerable, but has the purpose to estimate poverty indexes using a non-parametric technique based on a large number of boostrap samples.

All the indexes described so far are rich in terms of information summarised and stress different and equally relevant aspects of poverty risk. More precisely, Pritchett et al. (2000)'s or Chaudhuri (2003)'s index summarises downward and upward variability; the two FGT versions instead focus especially on the downward variability of income and account for different types of weights attached to extreme poverty, highlighting that the magnitude of the shock could be relevant in predicting future poverty. Calvo and Dercon (2005) consider the risk attitude important; and finally Dutta et al. (2011)'s measures are different from the others because they suggest that the current financial situation affects, in two opposite ways, the importance of the potential drops in income. It is not possible to distinguish a priori which is the best signal of poverty, since they favour different sides of the same phenomenon, or which predicts poverty more precisely when for instance a limited number of observations is available. Therefore we try to evaluate their predictive power empirically and classify them according to precision criteria conditional on information at hand.

\section{Data}

Vulnerability to poverty will be estimated using data drawn from the BHPS. The BHPS follows a representative sample of British individuals over the period 1991-2005; it was designed as an annual survey of each adult member for a nationally representative sample of about 5,000 households, making a total of approximately 10,000 individual interviews. The same individuals were re-interviewed in successive waves and, in case of split-off from the original household, all adults of the new household were also interviewed, preserving the representativeness of the British population. Additional sub-

\footnotetext{
${ }^{1}$ The values used in the empirical analysis for the additional parameters are the following: for CD rel $\alpha=0.5$, CD abs $\beta=0.5$, for DFM we use $\alpha=0.5$ and $\gamma=2$.
} 


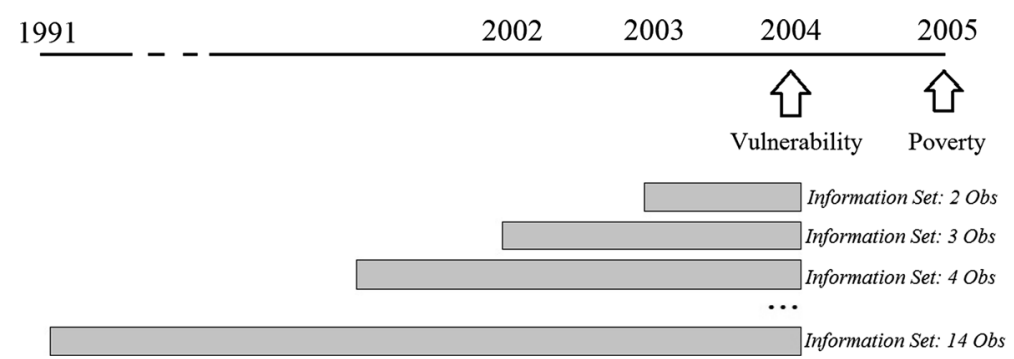

Fig. 1 Definition of information sets

samples were added in 1997 and 1999, respectively Scotland-Wales and Northern Ireland, to increase the relative small Scottish and Welsh samples size and to cover Norther Ireland properly, for a UK analysis rather than England only. In this analysis sample weights are not used, even if that is the conventional way to mitigate against potential attrition biases and new sub-samples effects. This is because the longitudinal weights supplied in the BHPS refer to a rather specific sample. The results therefore can be sensitive to the characteristics of the data used, especially to information about the net annual equivalized household income, provided for those households in which all eligible adults gave a full interview. The equivalence scale used is the square root of the household size and all values have been expressed in real terms (deflated to January 1998 prices).

As already mentioned, in order to compare indexes precision performances, according to different sets of information, we need a sufficiently large sample for the empirical analysis, with individuals or households observed in several occasions. In order to meet these requirements, we selected all households that were observed every year in the whole period. For each household, the poverty status that we consider to be anticipated is related to the last wave in which it participated, whereas to compute indexes we will exploit data immediately before the last year observed for selected number of waves. Figure 1 represents graphically how information sets are defined.

This strategies helps in looking always at the same households and focus only on the effect of the quantity of information used on predicting poverty. We consider, moreover, only households observed continuously every year for a more straightforward analysis without any complication related to the different timing of data collection, but we will relax this constraint in the robustness analysis.

The final sample is composed by 1,222 households, whose characteristics are summarized in Table 2. Missing information on education or region was retrieved from the previous waves. For Pritchett et al. (2000)'s and Chaudhuri (2003)'s approach, information about the age of the household head, the percentage of household members respectively with O-level of education or lower, A-level or equivalent and with a degree or higher education, is exploited as well as the percentage of children and earners.

\section{Empirical Strategy}

According to Chaudhuri (2003) and Pritchett et al. (2000)'s approach, if a panel dataset such as the BHPS is available, an income generating function can be defined as follows 
Table 2 Sample characteristics

\begin{tabular}{llr}
\hline UK-BHPS (1991-2005) & Obs & $\%$ \\
\hline Household Head's age & & \\
$\leq 44$ & 173049 & 14.00 \\
$\geq 45$ & & 86.00 \\
Poor & Mean & 13.09 \\
\hline & & SD \\
\hline Education & & 0.39 \\
\% O-level or lower in household & 0.20 & 0.42 \\
$\%$ A-level or equivalent in household & 0.11 & 0.31 \\
$\%$ Degree or higher in household & 0.40 & 0.26 \\
$\%$ Earners in household & 0.08 & 0.41 \\
$\%$ Children in household & 8,740 & 0.18 \\
Equivalent household annual disposable real income (f) in 2005 & 5,303 \\
\hline
\end{tabular}

Household annual disposable income has been deflated to January 1998 prices and equivalised using the square root of the household size. Poor identifies households whose equivalent annual disposable real income is lower than the $60 \%$ of the annual median value in 2005

$$
\begin{gathered}
y_{h, t}=y\left(X_{h}, \beta_{t}, \alpha_{h}, e_{h, t}\right) \\
v_{h, t}=E\left[p_{\alpha, h, t+1}\left(y_{h, t+1}\right) \mid F\left(y_{h, t+1} \mid X_{h}, \beta_{t}, \alpha_{h}, e_{h, t}\right)\right],
\end{gathered}
$$

where $X_{h}$ is a set of observable household characteristics, $\beta_{t}$ is a vector of parameters describing the economy at time $t, \alpha_{h}$ is an unobserved time-invariant household-level effect and $e_{h, t}$ represents any idiosyncratic shock that determines the variability of household income. This function will allow us to predict not only the income level at $t+1$, given the information up to time $t$, but also its variability in the period considered, using the residuals of the model specified.

According to this first method of assessing vulnerability as stated in expression 5, it is possible to estimate the conditional probability that the household income falls below the poverty line in the next period of time. Differently from Chaudhuri (2003), we use income as welfare measure, rather than consumption, simplifying the econometric issues related to predetermined, rather than strictly exogenous variables ${ }^{2}$ and the parameters are estimated using a fixed-effect model, where we control for education (with the percentage of household members having O-level or lower as reference category), a quadratic polynomial in (household head's) age, region and time dummies.

The econometric strategy is slightly different if the data considered are cross-sectional: it is not possible to follow households over time, but the heteroskedasticity in the data can be exploited to describe how the income variability changes according to some characteristics. This is the strategy used by Chaudhuri (2003) who estimates the parameters of the

\footnotetext{
${ }^{2}$ In his consumption generating function, Chaudhuri (2003) assumes that the elements of $X_{h, t}$ are contemporaneously uncorrelated with $e_{h, t}$ but allows for potential correlation between $X_{h, t}$ and lagged consumption shocks. If this is the case, the standard within-estimator cannot be used, that is the reason why Chaudhuri (2003) uses first differences of consumption and instruments the changes in the predetermined variables using lagged changes and levels of the same variables. In this case, if income is used rather than consumption, the correlation between $X_{h, t}$ and lagged shocks should not be an issue.
} 
specified model through a three-step feasible generalized least squares (FGLS) procedure, suggested by Amemiya (1977). This strategy imposes the assumption that households with similar characteristics are subjected to the same variability in income. In both crosssectional and panel analysis, normality is assumed to compute probabilities. As a consequence, in expression $6, \Phi(\cdot)$ denotes the cumulative density of the standard normal, $\hat{\mu}$ and $\hat{\sigma}$ are respectively the estimated expected equivalised disposable income at $t+1$ and the standard deviation;

$$
\hat{v}_{h, t}=\hat{p} r\left(\ln y_{h, t+1}<\ln z \mid \hat{\mu}_{\ln y_{h, t+1}}, \hat{\sigma}_{\ln y_{h, t+1}}^{2}\right)=\Phi\left(\frac{\ln z-\hat{\mu}_{\ln y_{h, t+1}}}{\hat{\sigma}_{\ln y_{h, t+1}}}\right) .
$$

For the FGT version of vulnerability to poverty (Christiaensen and Boisvert 2000), for Calvo and Dercon (2005) and Dutta et al. (2011) that do not explicitly define an income generating function as in the previous cases, we use as possible income values those already experienced by the household in the past, assuming that the data are informative about all the possible idiosyncratic shocks. In line with parametric estimates of vulnerability to poverty and differently from Celidoni (2013), probabilities attached to each income drop below the poverty line are computed assuming a normal distribution for each household, centered at the mean of the household annual disposable real income, standard deviation is computed using the same values. Therefore we will have in those cases (as in the panel analysis), one household vulnerability index, based on income values from 1991 to 2004 , to compare with poverty in $2005 .^{3}$

In order to understand which vulnerability measure can detect with more precision poor individuals in advance, we use the receiver operating characteristic curve (ROC), which can provide a summary of the degree to which vulnerability acts as a signal for poverty. This approach was originally used in the field of engineering or disease diagnosis, to measure how a given signal can detect an underlying condition. It has been then proposed also to assess the degree of overlapping between dimensions in the multidimensional poverty framework, see among others Madden (2011).

In this context income poverty in the last year observed $(t+1)$ is the underlying condition, whereas vulnerability indexes, computed on information up to time $t$, are the symptoms of poverty; by analyzing the ROC curves of each vulnerability measure is therefore possible to understand empirically which index is the most precise predictor of poverty.

To draw the ROC curve, we first define poor those households with equivalised disposable income in the last period observed lower than the traditional relative poverty threshold (60\% of the median equivalised income) and non-poor otherwise. Given the two groups, it is possible to understand, for each index, to what extent the distinction between vulnerable and non-vulnerable households produces the same partition based on the poverty status.

For each vulnerability threshold, we can distinguish the true positive (TP), i.e. those individuals that are vulnerable and poor, and the true negative (TN), i.e. those who are classified as non-poor and non-vulnerable. If the vulnerability threshold identifies as vulnerable someone who is not poor, he or she will be a false positive (FP), while false negative $(\mathrm{FN})$ is someone poor in income but non-vulnerable. The ROC curve exploits this classification to plot, on the vertical axis, the sensitivity or TP rate, TP/(TP $+\mathrm{FN})$, against 1-the specificity or $\mathrm{TN}$ rate, $1-\mathrm{TN} /(\mathrm{FP}+\mathrm{TN})$, on the horizontal axis, for all possible

\footnotetext{
${ }^{3}$ We recall that, differently from all the other vulnerability indexes, the cross-sectional approach (Chaudhuri 2003) exploits only the information available in 2004.
} 
Fig. 2 The ROC curve

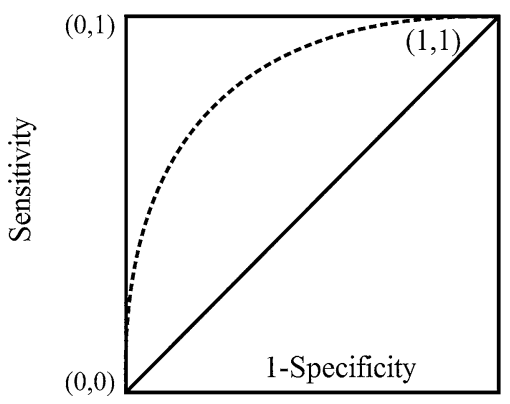

vulnerability thresholds. The higher the correlation between vulnerability and poverty (the higher will be the sensitivity and the specificity), the more vulnerability acts as a predictor for poverty and, in graphical terms (Fig. 2), the nearer will be the curve to the point $(0,1)$. For a more straightforward and intuitive summary of this correlation, we report the area under the ROC curve: the higher is this area the better the signaling.

Even if the area under the ROC curve is a measure of association specifically designed to deal with dichotomous variables, other two alternative criteria are used: the Pearson and the Spearman correlation coefficients. While the ROC curve is appropriate for binary variables, the correlation coefficients reflect the correlation between individuals across the complete distribution of vulnerability and income. Especially, the Pearson coefficient estimates a linear dependence, while the Spearman correlation coefficient is a non-parametric measure of statistical dependence less sensitive to strong outliers that are in the tails.

\section{Results}

Before looking at vulnerability indexes, we will briefly report some summary statistics about poverty in our sample in order to highlight those features that might affect our results about identification precision. Among the selected households, about the $59 \%$ never experienced poverty between 1991 and 2005. The remaining $41 \%$, even if on average faces poverty 5 times over a period of 15 years, is not long-term poor. We provide in Fig. 3 the percentage of households experiencing different numbers of poverty episodes. As we can see, they concentrate especially on one or two poverty episodes, suggesting the transitory nature of the phenomenon that we observe in our selected sample.

We report in Table 3 a summary of the discussed vulnerability indexes, especially the mean value and the standard deviation for the two categories of households, poor and non poor. It is possible to notice that, for every measure, poor households are, on average, more vulnerable than the non poor. The ratio of vulnerability between the two categories is different among indexes and depends also on their functional form. For instance in the UK, according to Pritchett et al. (2000)'s index, poor households are twice more vulnerable in terms of probability, than those who turned out to be non poor, while for the index proposed by Dutta et al. (2011), when the low current living standard exacerbates the potential drops in welfare, this ratio is much higher.

We now focus on Table 4 to assess whether the index predictive power depends on the available data. We will comment our results according to the area under the ROC curve, our preferred precision criterion. According to previous findings, for every information set, we can distinguish two groups of measures, those with an area sistematically larger than 0.8 , that 


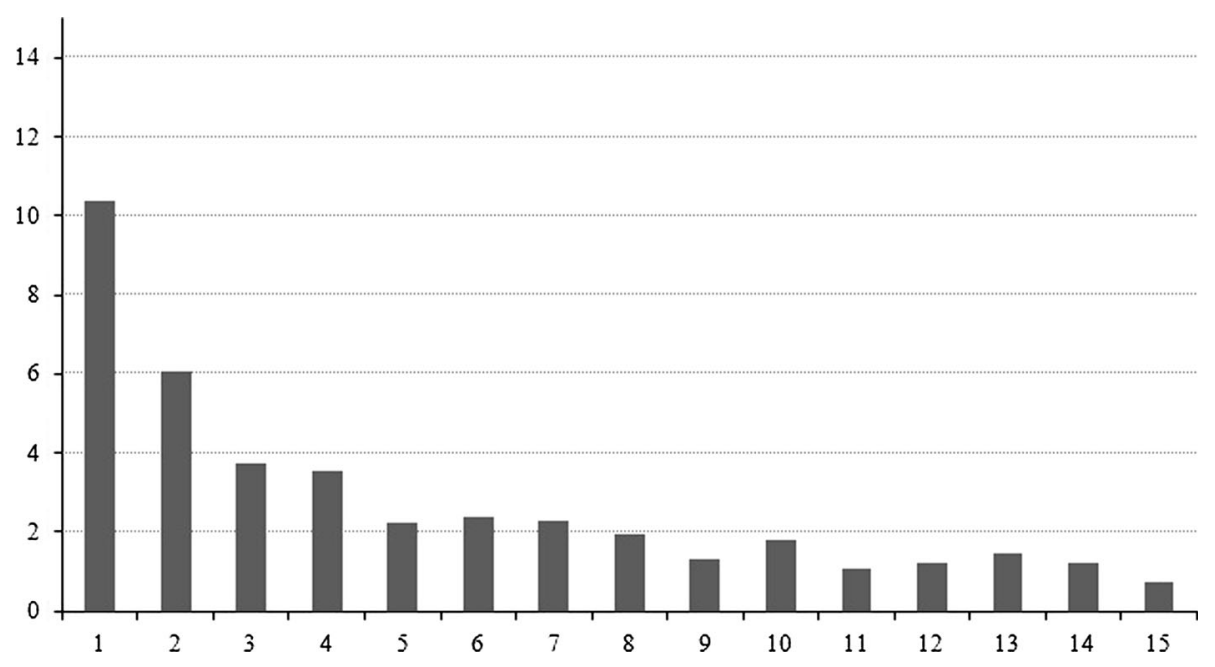

Fig. 3 Number of poverty episodes, \% of households

Table 3 Vulnerability to Poverty Indexes

\begin{tabular}{lllll}
\hline Index & \multicolumn{2}{l}{ Non Poor } & & \multicolumn{2}{l}{ Poor } \\
\cline { 2 - 3 } & Mean & SD & Mean & SD \\
\hline PC & 0.0501 & 0.0972 & 0.1035 & 0.1360 \\
C & 0.0444 & 0.0985 & 0.1245 & 0.1598 \\
FGT1 & 0.0150 & 0.0384 & 0.0774 & 0.0901 \\
FGT2 & 0.0059 & 0.0186 & 0.0303 & 0.0548 \\
CD rel & 0.1238 & 0.0852 & 0.1555 & 0.1072 \\
CD abs & 0.0128 & 0.0334 & 0.0661 & 0.0804 \\
DFM1/10 & 0.5102 & 1.2080 & 0.6619 & 0.9781 \\
DFM2/10 & 0.0031 & 0.0403 & 7.0190 & $8.76 \times 10$ \\
\hline
\end{tabular}

can be labelled as high-performers, and those with lower values, the low-performers. ${ }^{4}$ Looking at the ROC area point estimates, the indexes that belong to the high-performers group are the FGT versions of vulnerability, the Calvo and Dercon (2005)'s index that accounts for the absolute risk sensitivity, and the second version of Dutta et al. (2011)'s measure of poverty risk. In particular, the second version of Dutta et al. (2011)'index in general records the largest estimated value for the area under the ROC curve.

While the between groups classification is stable over different information sets, some indexes show statistically different point estimates of the area between the richest (14 yearly observations) and the poorest (only 2 yearly observations) information set. We provide a graphical representation of those differences in Fig. 4. Looking at the Figure in fact it is possible to notice that, comparing results between the two information sets, the

${ }_{4}$ In Table 4, 5 and 6, high-performers indexes are reported in italics. 


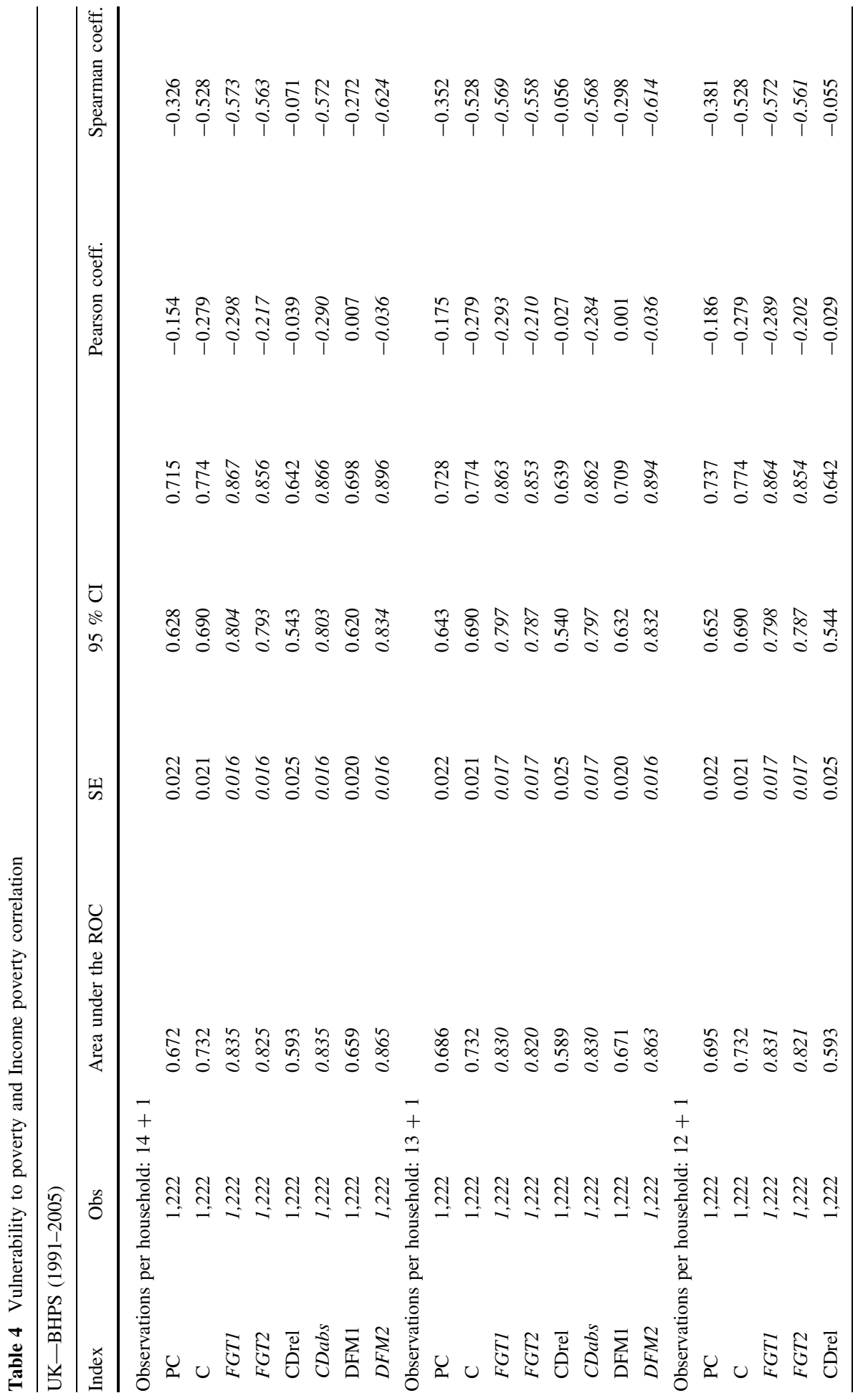




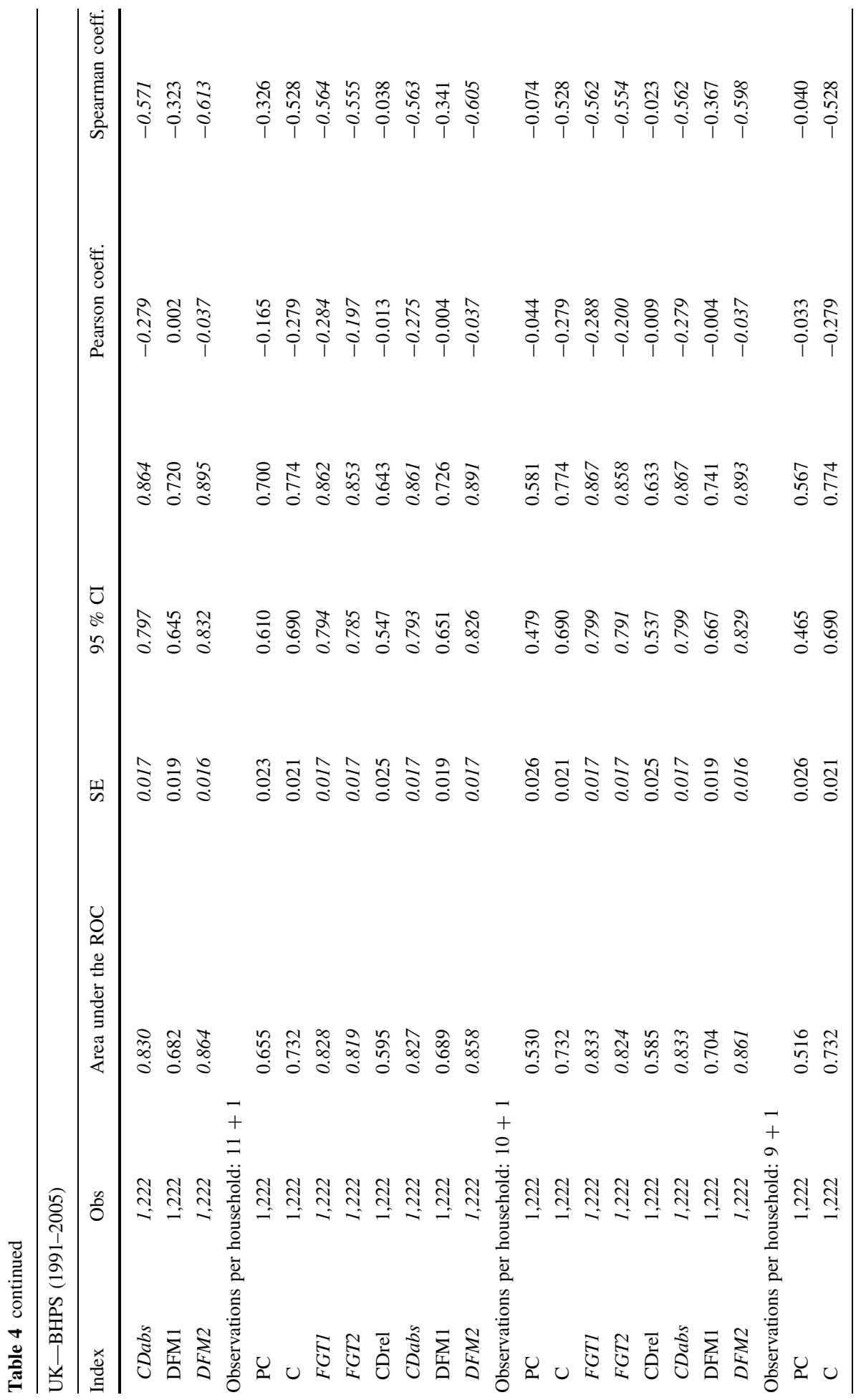




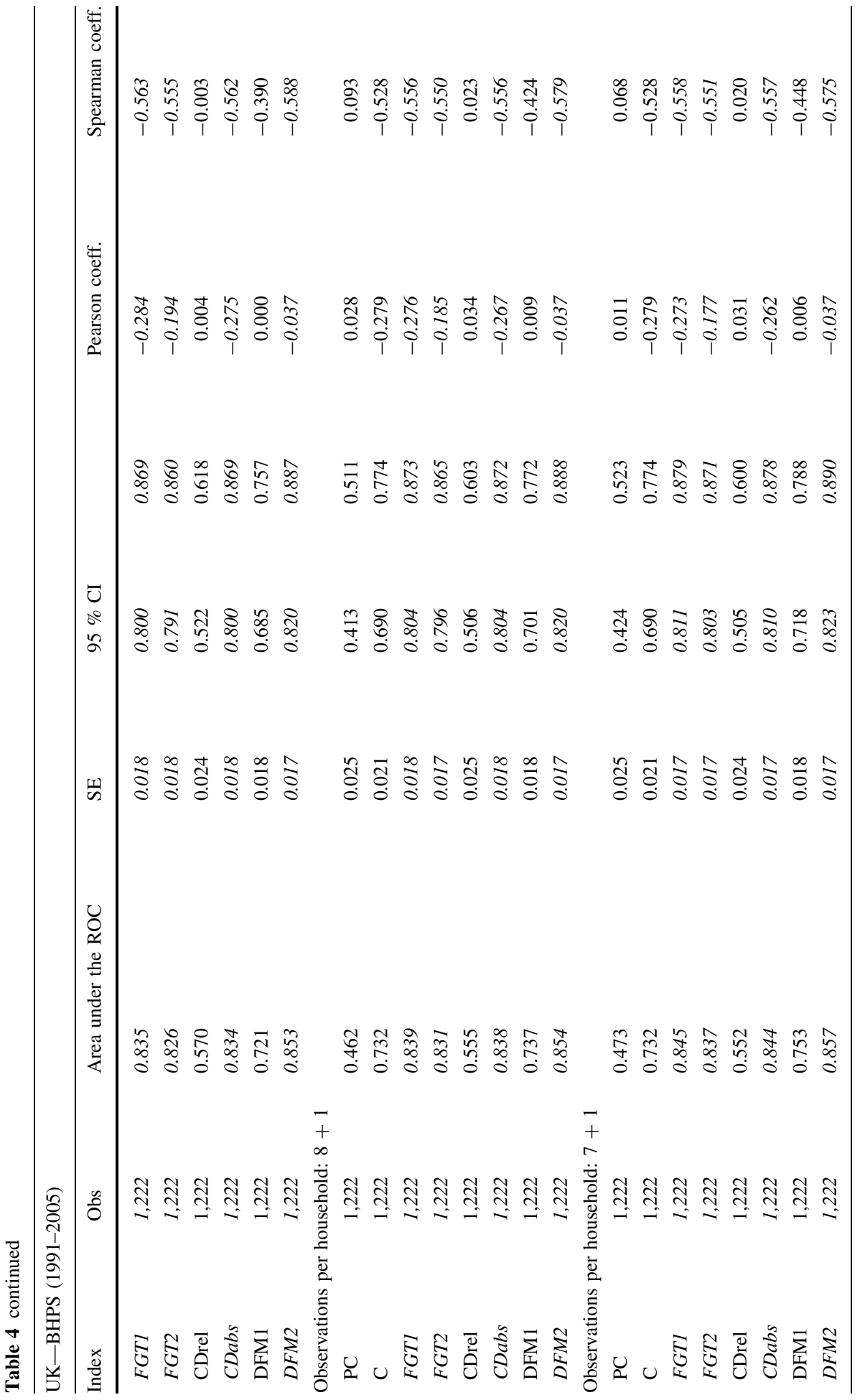




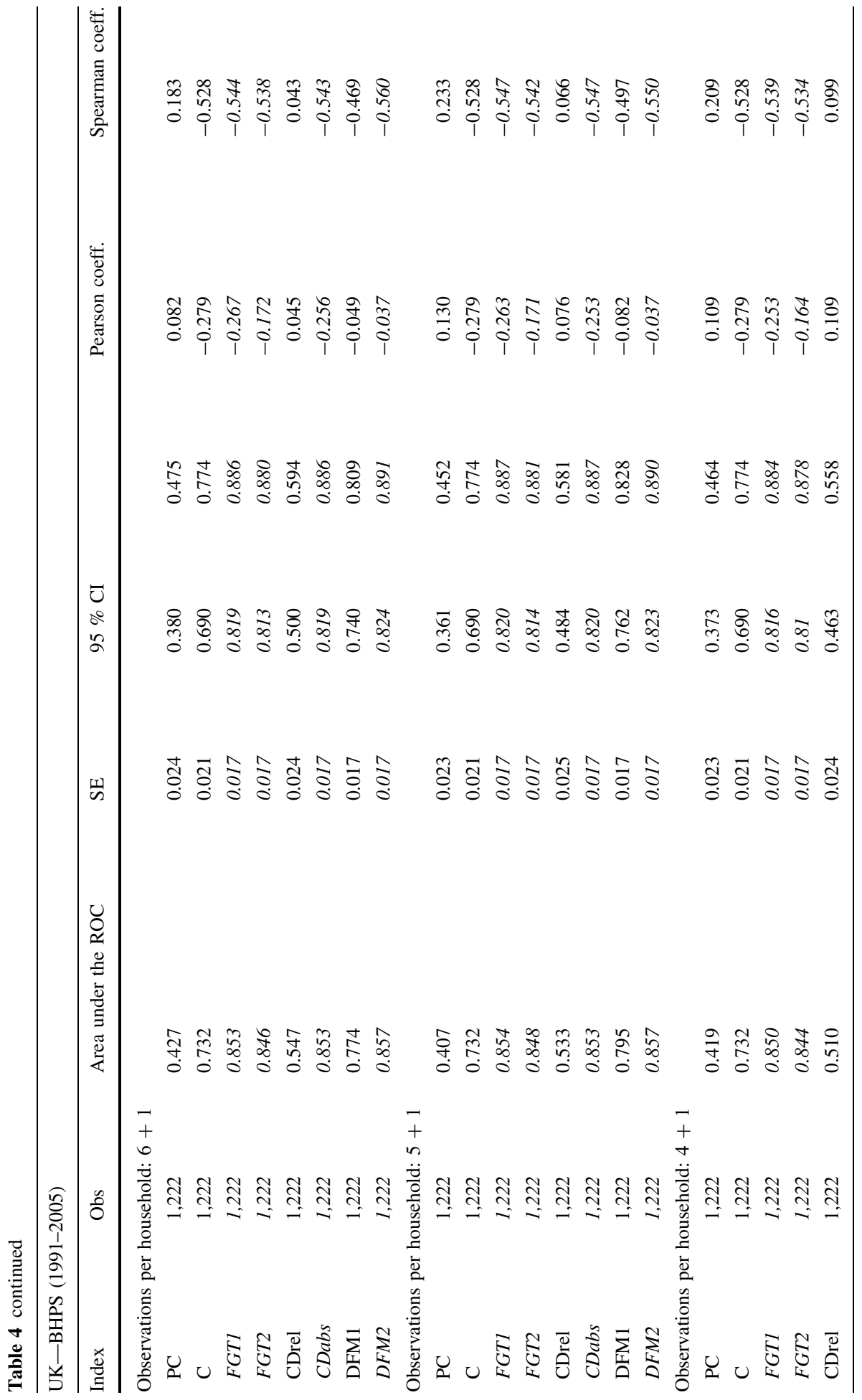




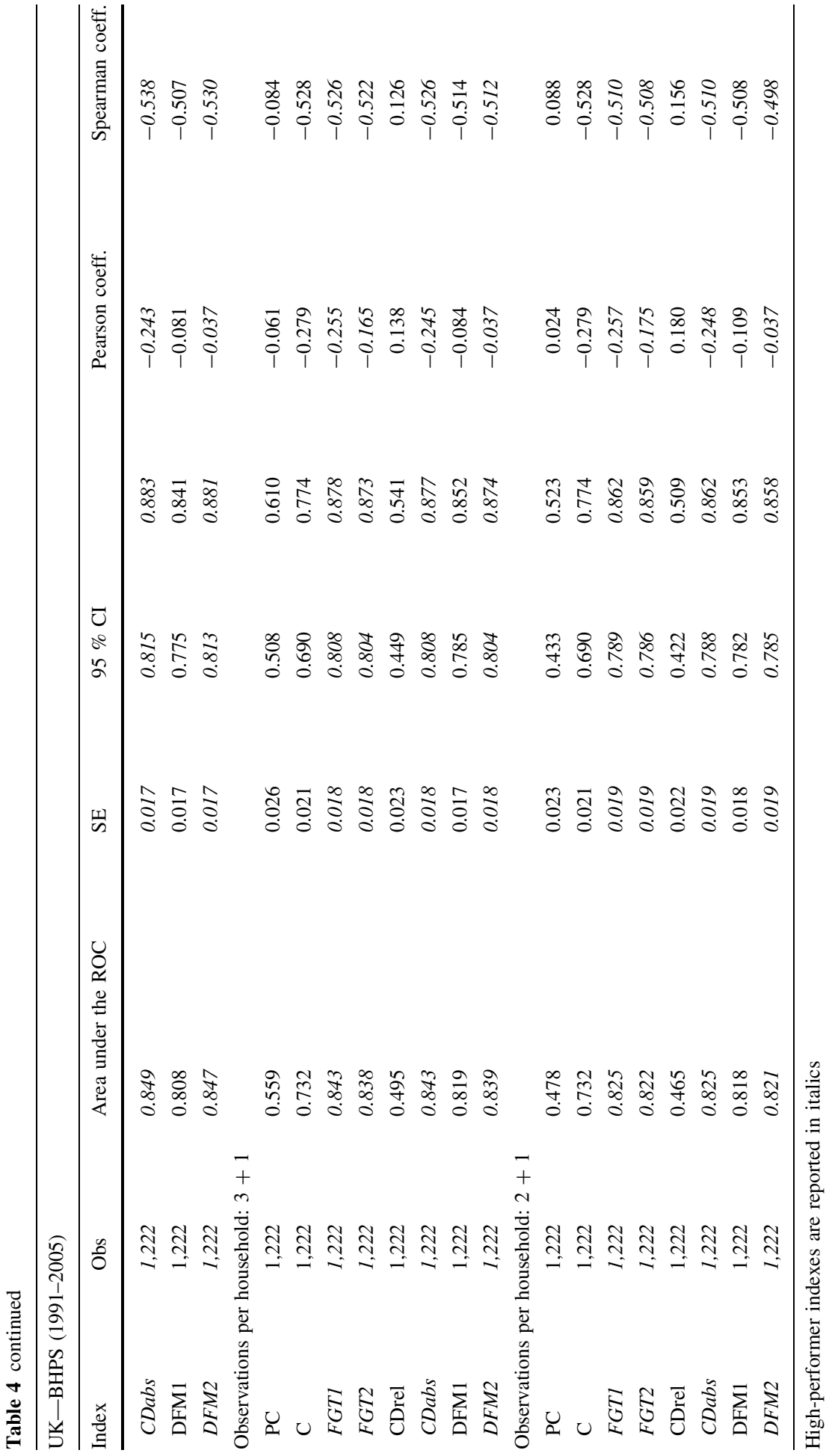




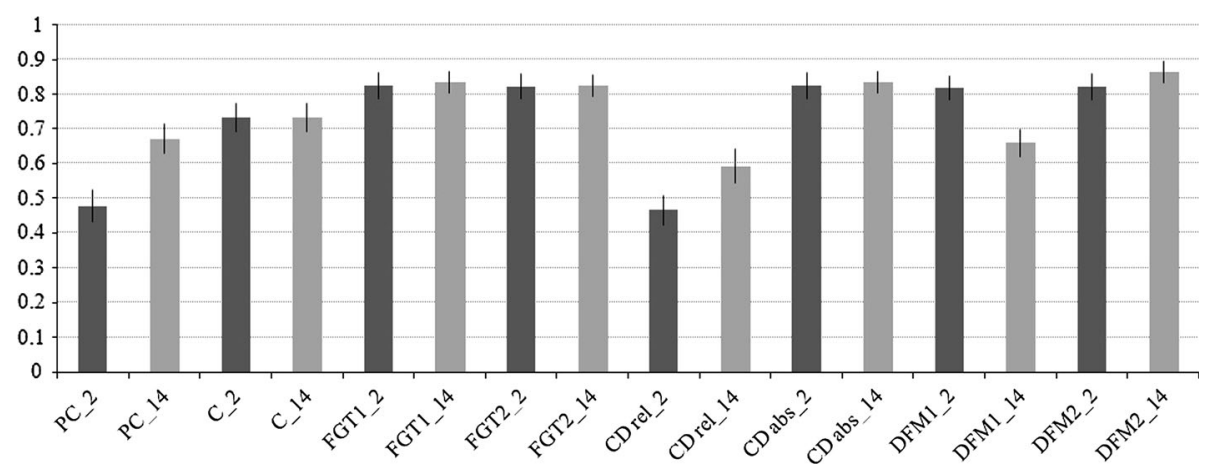

Fig. 4 Area under the ROC curve, by different information sets

$95 \%$ confidence intervals overlap for Christiaensen and Boisvert (2000)'s, the absolute version of Calvo and Dercon (2005)'s, the second version of Dutta et al. (2011)'s indexes and, by construction, for the Chaudhuri (2003)'s measure of vulnerability to poverty. Pritchett et al. (2000)'s measure, the relative version of Calvo and Dercon (2005)'s index and Dutta et al. (2011)'s first version of vulnerability to poverty change their predictive power when more information is used. Especially, with more data at hand, Pritchett et al. (2000)'s and Calvo and Dercon (2005)'s measures improve their performances while Dutta et al. (2011)'s DFM1 index worsens it. We recall that DFM1 incorporates the idea of worse consequences in terms of vulnerability for those with higher current living standard, which seems to be a reasonably weaker predictor of future poverty compared to other indexes and more information at hand highlights that.

Our first results show the role, from the identification point of view, of a long longitudinal dimension to be exploited especially when using particular vulnerability indexes. In line with the literature, therefore we find that a rich longitudinal perspective is an essential ingredient for policy formulation (Jenkins 2007), especially if we focus on targeting based for instance on panel analysis.

The area under the ROC curve, used so far, can be seen as a criterion for the overall signal precision: all the FP-FN combinations are chosen by varying the threshold that divides vulnerable and non vulnerable individuals. However, in this context, the two types of errors could have a different relevance for assessing the signal precision: identifying as non-vulnerable households that will be poor is worse than defining as vulnerable someone who will not be poor.

Both errors anyhow cannot be reduced at the same time, so that if few FNs are prefererred, a higher error in terms of false positive has to be tollerated and viceversa. A possible strategy to take into account differently the relevance of the two errors, is to choose a specific, high and fixed level of sensitivity and classify the vulnerability measures in terms of specificity. Raking our indexes according to the latter will give us an idea of the identification precision when we tolerate only a certain percentage of undercoverage (or type I error). The rank based on different fixed levels of sensitivity, might not correspond to the overall performance of the indexes among different information sets.

In Table 5, we report therefore specificity rates given fixed levels of sensitivity, i.e. 85, 80 and $75 \%$, for the richest and the poorest information set. Especially, the higher the specificity, for a certain sensitivity level, the lower the fraction of false positive and the better the signal. Looking at specificity levels, it can be noticed the same distinction 
Table 5 Specificity for fixed sensitivity levels (\%)

\begin{tabular}{|c|c|c|c|c|c|c|}
\hline \multirow{2}{*}{$\frac{\text { Index }}{\text { Sensitivity }}$} & \multicolumn{3}{|c|}{ Observations per household: $14+1$} & \multicolumn{3}{|c|}{ Observations per household: $2+1$} \\
\hline & 85 & 80 & 75 & 85 & 80 & 75 \\
\hline PC & 34.93 & 45.57 & 50.56 & 34.28 & 34.28 & 34.28 \\
\hline $\mathrm{C}$ & 46.52 & 58.76 & 65.16 & 46.52 & 58.76 & 65.16 \\
\hline$F G T 1$ & 71.85 & 75.89 & 80.79 & 90.49 & 90.49 & 90.49 \\
\hline$F G T 2$ & 72.13 & 75.05 & 78.53 & 90.49 & 90.49 & 90.49 \\
\hline CD rel & 17.14 & 21.94 & 34.84 & 20.06 & 25.80 & 27.68 \\
\hline$C D a b s$ & 71.94 & 75.80 & 80.89 & 90.49 & 90.49 & 90.49 \\
\hline DFM1 & 41.05 & 46.80 & 51.69 & 86.16 & 86.16 & 87.10 \\
\hline DFM2 & 77.50 & 82.49 & 85.78 & 92.00 & 92.00 & 92.00 \\
\hline
\end{tabular}

High-performer indexes are reported in italics

Table 6 Vulnerability to poverty and Income poverty correlation

\begin{tabular}{|c|c|c|c|c|c|c|c|}
\hline Index & Obs & Area under the ROC & SE & \multicolumn{2}{|c|}{$95 \% \mathrm{CI}$} & Pearson coeff. & Spearman coeff. \\
\hline \multicolumn{8}{|c|}{ Observations per household: $7+1$ most recent } \\
\hline $\mathrm{PC}$ & 1,222 & 0.473 & 0.025 & 0.424 & 0.523 & 0.011 & 0.068 \\
\hline $\mathrm{C}$ & 1,222 & 0.732 & 0.021 & 0.690 & 0.774 & -0.279 & -0.528 \\
\hline$F G T 1$ & 1,222 & 0.845 & 0.017 & 0.811 & 0.879 & -0.273 & -0.558 \\
\hline$F G T 2$ & 1,222 & 0.837 & 0.017 & 0.803 & 0.871 & -0.177 & -0.551 \\
\hline CDrel & 1,222 & 0.552 & 0.024 & 0.505 & 0.600 & 0.031 & 0.020 \\
\hline CDabs & 1,222 & 0.844 & 0.017 & 0.810 & 0.878 & -0.262 & -0.557 \\
\hline DFM1 & 1,222 & 0.753 & 0.018 & 0.718 & 0.788 & 0.006 & -0.448 \\
\hline$D F M 2$ & 1,222 & 0.857 & 0.017 & 0.823 & 0.890 & -0.037 & -0.575 \\
\hline \multicolumn{8}{|c|}{ Observations per household: $7+1$ randomly selected } \\
\hline PC & 1,222 & 0.567 & 0.025 & 0.518 & 0.615 & -0.053 & -0.135 \\
\hline $\mathrm{C}$ & 1,222 & 0.731 & 0.021 & 0.689 & 0.773 & -0.285 & -0.520 \\
\hline$F G T 1$ & 1,222 & 0.809 & 0.019 & 0.773 & 0.845 & -0.280 & -0.527 \\
\hline$F G T 2$ & 1,222 & 0.800 & 0.018 & 0.764 & 0.836 & -0.193 & -0.519 \\
\hline CDrel & 1,222 & 0.503 & 0.024 & 0.456 & 0.551 & 0.062 & 0.048 \\
\hline CDabs & 1,222 & 0.808 & 0.019 & 0.772 & 0.845 & -0.271 & -0.526 \\
\hline DFM1 & 1,222 & 0.692 & 0.019 & 0.655 & 0.730 & 0.013 & -0.303 \\
\hline$D F M 2$ & 1,222 & 0.831 & 0.018 & 0.796 & 0.867 & -0.081 & -0.578 \\
\hline
\end{tabular}

High-performer indexes are reported in italics

between high- and low-performers also in this case, with the second version of Dutta et al. (2011)'s measure of poverty risk recording the highest specificity rate.

In order to understand the role of the different timing of data collection in this framework, we propose a robustness analysis. All the information sets used so far, considered the most recent income $n$ observations, where $n$ goes from 2 to 14 . But it could be the case that policy-makers have discontinuous and not always updated information at hand to exploit for targeting purposes. The role of regular and updated information in predicting poverty can be observed in Table 6, where we show results in case we have regular observations compared to randomly chosen, among the 14 available, irregular 
observations. To have enough timing variability, we consider the case in which we have half of the available income data per household at our disposal. ${ }^{5}$ In Table 6 looking at confidence intervals, it is possible to notice that results either remain stable or, as expected, improve the more recent and regular is the information exploited.

\section{Conclusions}

According to the chosen correlation criterion, the ROC curve, which is specifically designed for binary variables, we find that Celidoni (2013)'s distinction between high-and low-performers does not vary according to the quantity of information at hand, but some indexes are more responsive than other to different information sets: Pritchett et al. (2000)'s measure, the relative version of Calvo and Dercon (2005)'s index and Dutta et al. (2011)'s first version of vulnerability to poverty change their predictive power when more information is used. Especially, with more available data, Pritchett et al. (2000)'s and Calvo and Dercon (2005)'s measures improve their performances while Dutta et al. (2011)'s DFM1 index worsens it. This highlights that especially high-performer vulnerability measures can be used to support the design of better risk-management and antipoverty policies also in presence of a limited longitudinal data dimension. This facilitates the use of vulnerability indexes to complement the standard poverty risk analysis and provide more insights especially in case of crisis and financial downturns, when the success of generally used strategies for recovery is more unlikely.

Vulnerability measures in fact capture individuals who are exposed to poverty risk and can be used to understand which are the characteristics of the future poor. This ex ante information, by describing the different types of risk, could help in designing better protection policies and prevent households and individuals from experiencing severe welfare losses.

As already emphasised in the literature risk is quite different in size, likelihood, and frequency over time and different features correspond to different implications for the ability to cope with them as well as for policy purposes (Dercon 2001). Also Morduch (2000) says that it is important considering some of the patterns related to risk, since they have quite different impacts on the ability to cope with them for individuals, households, communities, and other institutions.

Vulnerability to poverty can be used therefore to highlight for instance, whether poverty risk increased because there are more chances of facing adverse events such as unemployement, sickness or disability, or/and because negative shocks become more severe or/ and more unpredictable, see Celidoni (2014) for a proposed decomposition that highlights these three contributing factors of poverty risk. This decomposition as well as similar empirical analysis (e.g. Pritchett et al. 2000) might provide additional important information about why individuals are not able to properly insure against negative shocks that lead to poverty and help policy-makers to improve policies design.

\section{References}

Amemiya, T. (1977). The maximum likelihood estimator and the non-linear three stage least squares estimator in the general nonlinear simultaneous equation model. Econometrica, 45, 955-968.

\footnotetext{
${ }^{5}$ Results do not change whether we consider different information sets.
} 
Atkinson, A. B. (1995). On targeting social security: Theory and western experience with family benefits. In D. Van de Walle \& K. Nead (Eds.), Public spending and the poor: Theory and evidence (pp. 25-68). Washington, D.C.: The Johns Hopkins University Press for the World Bank.

Azam, M., \& Imai, K. (2009). Vulnerability and poverty in Bangladesh. Discussion Paper Series 0905, the University of Manchester, The School of Economics.

Bandyopadhyay, S., \& Cowell, F. (2007). Modelling vulnerability in the UK. LSE STICERD Research Paper 89.

Calvo, C., \& Dercon, S. (2005). Measuring individual vulnerability. Economics Series Working Papers 229, University of Oxford, Department of Economics.

Celidoni, M. (2013). Vulnerability to poverty: An empirical comparison of alternative measures. Applied Economics, 45, 1493-1506.

Celidoni, M. (2014). Decomposing vulnerability to poverty. Review of Income and Wealth (forthcoming).

Chaudhuri, S. (2003). Assessing household vulnerability to poverty: Concepts, empirical methods and illustrative examples. Mimeo, Columbia University.

Chaudhuri, S., Jalan, J., \& Suryahadi, A. (2002). Assessing household vulnerability to poverty from crosssectional data: A methodology and estimates from Indonesia. Discussion paper 0102-52, Columbia University, Department of Economics.

Christiaensen, L., \& Boisvert, R. (2000). On measuring household food vulnerability: Case evidence from northern Mali. Working papers. Department of Agricultural, Resource and Managerial Economics, Cornell University, NY.

Dercon, S. (2001). Assessing vulnerability to poverty. Mimeo, Jesus College, Oxford and Centre for the Study of African Economies (CSAE), Department of Economics, Oxford University.

Dercon, S. (2006). Vulnerability: A micro perspective. QEH working papers 149, 2006.

Dutta, I., Foster, J., \& Mishra, A. (2011). On measuring vulnerability to poverty. Social Choice and Welfare, 37, 743-761.

Foster, J., Greer, J., \& Thorbecke, E. (1984). A class of decomposable poverty measures. Econometrica, 52, 761-766.

Gaiha, R., \& Imai, K. (2008). Measuring vulnerability and poverty: Estimates for rural India. Working paper 2008/40. World Institute for Development Economic Research (UNU-WIDER).

Gaiha, R., Imai, K., \& Kang, W. (2011). Vulnerability and poverty dynamics in Vietnam. Applied Economics, 43, 3603-3618.

Gerry, C., \& Li, C. (2010). Consumption smoothing and vulnerability in Russia. Applied Economics, 42, 1995-2007.

Hoddinott, J., \& Quisumbing, A. R. (2003). Methods for microeconometric risk and vulnerability assessments. Social Protection Discussion Paper Series 0324, The World Bank.

Imai K., Wang, X., \& Kang, W. (2009). Poverty and vulnerability in rural China: Effects of taxation. Discussion Paper Series 0913, The University of Manchester, The School of Economics.

Jalan J., \& Ravaillon, M. (1998). Determinants of transient and chronic poverty: Evidence from rural China. Working paper no. 1936, World Bank Policy Research.

Jamal, H. (2009). Assessing vulnerability to poverty: Evidence from Pakistan. Research report no. 80, Social Policy and Development Centre (SPDC).

Jenkins, S. (2007). New directions in the analysis of inequality and poverty. Technical report, IZA discussion papers 2814.

Jha, R., Dang, T., \& Sharma, K. (2009). Vulnerability to poverty in Fiji. International Journal of Applied Econometrics and Quantitative Studies, 6, 51-68.

Kamanou, G., \& Morduch, J. (2002). Measuring vulnerability to poverty. Working paper 2002/58, World Institute for Development Economic Research (UNU-WIDER).

Ligon, E., \& Schechter, L. (2003). Measuring vulnerability. Economic Journal, 113, C95-C102.

Madden, D. (2011) Health and income poverty in Ireland, 2003-2006. Journal of Economic Inequality, 9, 23-33.

Morduch, J. (2000). Between the state and the market: Can informal insurance patch the safety net? World Bank Research Observer, 14, 187-207.

Muller, C., \& Bibi, S. (2010). Refining targeting against poverty evidence from Tunisia. Oxford Bulleting of Economics and Statistics, 72, 381-410.

OECD. (2011). Divided we stand: Why inequality keeps rising. Technical report, OECD, The Organisation for Economic Co-operation and Development.

Osberg, L. (1998). Economic insecurity. SPRC Discussion paper 88, University of New South Wales, Social Policy Research Centre.

Osberg, L. (2010). Measuring economic insecurity and vulnerability as part of economic well-being: Concepts and context. In IARIW 31st general conference. St. Gallen, Switzerland. 
Pritchett, L., Suryahadi, A., \& Sumarto, S. (2000). Quantifying vulnerability to poverty: A proposed measure applied to Indonesia. Working paper WPS 2437, The World Bank.

Skoufias, E., \& Quisumbing, A. R. (2003). Consumption insurance and vulnerability to poverty: A synthesis of the evidence from Bangladesh, Ethiopia, Mali, Mexico and Russia. FCND Discussion paper 155, International Food Policy Research Institute.

The World Bank (2011). Measuring vulnerability. Available at http://web.worldbank.org/WBSITE/ EXTERNAL/TOPICS/EXTPOVERTY/EXTPA/0,,contentMDK:20238993 menuPK: $492141 \sim$ pagePK:148956 piPK:216618 theSitePK:430367,00.html. Accessed 10 Feb 2011.

Zhang, Y., \& Guanghua, W. (2008). Can we predict vulnerability to poverty? WIDER research paper 2008/82. 